\title{
Density of states anomalies in hybrid superconductor-ferromagnet-normal metal structures
}

\author{
A. A. Golubov, ${ }^{1}$ M. Yu. Kupriyanov, ${ }^{2}$ and M. Siege ${ }^{3}$ \\ ${ }^{1}$ Faculty of Science and Technology, University of Twente, The Netherlands \\ ${ }^{2}$ Nuclear Physics Institute, Moscow State University, 119992 Moscow, Russia \\ ${ }^{3}$ Institute for Micro and Nanoelectronic Systems, \\ Karlsruhe University, Hertzstrasse 16, D-76187 Karlsruhe, Germany
}

(Dated: 20 January 2005)

\begin{abstract}
The results of calculations of the spatially-resolved density of states (DoS) in an $\mathrm{S}(\mathrm{F} / \mathrm{N})$ bilayer are presented ( $\mathrm{S}$ is a superconductor, $\mathrm{F}$ is a metallic ferromagnet, $\mathrm{N}$ is a normal metal) within quasiclassical theory in the dirty limit. Analytical solutions are obtained in the case of thin $\mathrm{F}$, $\mathrm{N}$ layers which demonstrate the peculiar features of DoS in this system. The dependencies of the minigap and the DoS peak positions on the exchange energy and parameters of the layers are studied numerically.
\end{abstract}

PACS numbers: 74.50.+r, 74.80.Dm, 75.30.Et

In the past few years there was a noticeable interest to the Josephson junctions with ferromagnetic barriers due to possibility to realize the $\pi$-junctions having the phase difference $\pi$ in the ground state. The $\pi$-states in SFS junctions were first predicted by ${ }^{2.3 .4}$ and realized experimentally by Ryazanov et al ${ }^{5.6}$ in $\mathrm{Nb} / \mathrm{CuNi} / \mathrm{Nb}$ structures and later by other groups $7.8,9,10,11$ using different ferromagnetic barriers. These experiments stimulated further theoretical activity (see 1 for the review). In particular, Josephson structures composed from arrays of $0-$ and $\pi-$ Josephson junctions should exhibit extraordinary characteristics 12.13 . Such arrays were recently realized in zig-zag HTS/LTS structures 14 .

The purpose of the present paper is to study spatially resolved electronic density of states (DoS) in the structure of S(FN)type, consisting of a bulk superconductor with ferromagnetic and normal layers on the top of it, which is a generic system for 0 - and $\pi$-junctions connected in parallel.

DoS in SF bilayers (a ferromagnet coupled to a superconductor) was studied quite extensively before. Two new features were predicted compared to SN systems: spin splitting and spatial oscillations of DoS in a ferromagnet $16.17 .18 \cdot 19 \cdot 20.21 .22$. The effect of spatial oscillations was quite extensively discussed in the theoretical literature in different models $\frac{16.17 .18 .19}{19}$ and observed experimentally 15 . This effect is closely related to $0-\pi$ transitions. The effect of splitting is relevant for thin ferromagnetic layers and was studied theoretically in ${ }^{20.21}$. In the present work we discuss an interplay between the oscillations and splitting in a more complex $\mathrm{S}(\mathrm{F} / \mathrm{N})$ structure.

The geometry of the structure is shown on Fig.1. We assume that the dirty limit conditions are fulfilled in all metals, $\mathrm{F}$ is a weak monodomain ferromagnet with the exchange energy $H$ much smaller than the Fermi energy and the interfaces are not magnetically active. In this case spin dependent corrections to the resistivities can be neglected and the $\mathrm{S}(\mathrm{F} / \mathrm{N})$ structure is described by

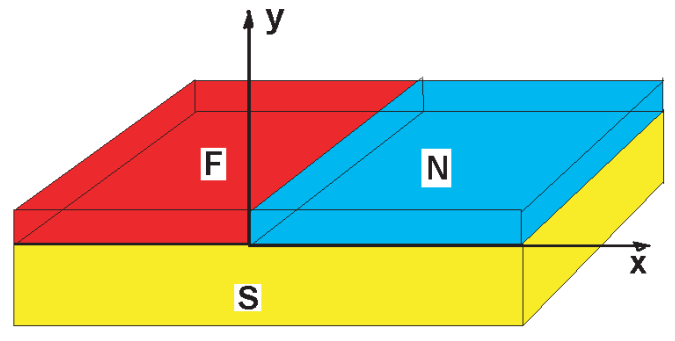

FIG. 1: The geometry of the structure.

the following spin independent suppression parameters

$$
\begin{gathered}
\gamma_{B F}=R_{B F} \mathcal{A}_{B F} / \rho_{F} \xi_{F}, \quad \gamma_{F}=\rho_{S} \xi_{S} / \rho_{F} \xi_{F}, \\
\gamma_{B N}=R_{B N} \mathcal{A}_{B N} / \rho_{N} \xi_{N}, \quad \gamma_{N}=\rho_{S} \xi_{S} / \rho_{N} \xi_{N}, \\
\gamma_{B}=R_{B} \mathcal{A}_{B} / \rho_{N} \xi_{N}, \quad \gamma=\rho_{F} \xi_{F} / \rho_{N} \xi_{N} .
\end{gathered}
$$

Here $R_{B F}, R_{B N}, R_{B}$ are the specific resistivities of the $\mathrm{SF}, \mathrm{SN}$ and NF interfaces respectively; $\rho_{S, F, N}, D_{S, F, N}$ and $\xi_{S, F, N}$ are the resistivities, the diffusion constants and the coherence lengths of the S, F and $\mathrm{N}$ layer and the coherence lengths, where $\xi_{S, F, N}=\sqrt{D_{S, F, N} / 2 \pi T_{c}}$ and $T_{c}$ is the critical temperature of the superconductor.

Under the above assumptions the problem can be solved in the framework of the Usadel equations ${ }^{23}$. To simplify it further we assume that $\mathrm{S}$ is a bulk superconductor and $\gamma_{N} \ll \gamma_{B N}, \quad \gamma_{F} \ll \gamma_{B F}$ so that the rigid boundary conditions

$$
F_{S}=\frac{\Delta}{\sqrt{\omega^{2}+\Delta^{2}}}, \quad G_{S}=\frac{\omega}{\sqrt{\omega^{2}+\Delta^{2}}}
$$


are valid for superconductor. Here $\Delta$ is the magnitude of the order parameter in S electrode, $F_{S}$ and $G_{S}$ are the Green's functions, $\omega=\pi T(2 n+1)$ are the Matsubara frequencies.

Let us choose the $x, y$ axes as shown in Fig.1 and use the $\theta$ parametrization $G=\cos \theta, F=\sin \theta$, then the Usadel equations have the form

$$
\xi_{F, N}^{2} \frac{\pi T_{c}}{\widetilde{\omega}}\left\{\frac{\partial^{2}}{\partial x^{2}} \theta_{F, N}+\frac{\partial^{2}}{\partial y^{2}} \theta_{F, N}\right\}-\sin \theta_{F, N}=0,
$$

where $\widetilde{\omega}=\omega+i H$ in $\mathrm{F}$ and $\widetilde{\omega}=\omega$ in $\mathrm{N}$.

The boundary conditions at the SF $(y=0,-\infty<x \leq$ $0)$, $\mathrm{SN}(y=0,0 \leq x<\infty)$ and $\mathrm{FN}\left(x=0,0 \leq y \leq d_{F}\right.$, $\left.d_{N}\right)$ interfaces have the form 24

$$
\begin{gathered}
\gamma_{B(F, N)} \xi_{F, N} \frac{\partial}{\partial y} \theta_{F, N}=-\sin \left(\theta_{S}-\theta_{F, N}\right), y=0 \\
\gamma_{B} \xi_{F} \frac{\partial}{\partial x} \theta_{F}=\sin \left(\theta_{N}-\theta_{F}\right), x=0,0 \leq y \leq d_{F}, d_{N} \\
\xi_{N} \frac{\partial}{\partial x} \theta_{N}=\gamma \xi_{F} \frac{\partial}{\partial x} \theta_{F}, x=0,0 \leq y \leq d_{F}, d_{N}
\end{gathered}
$$

where $\sin \theta_{S}=\Delta / \sqrt{\omega^{2}+\Delta^{2}}$ and $\cos \theta_{S}=\omega / \sqrt{\omega^{2}+\Delta^{2}}$. At the free interfaces the boundary conditions are

$$
\begin{gathered}
\frac{\partial}{\partial y} \theta_{F, N}=0, \quad y=d_{F, N} \\
\frac{\partial}{\partial x} \theta_{F, N}=0, \quad 0 \leq y \leq d_{F}, \quad x \rightarrow \mp \infty
\end{gathered}
$$

We will consider the limit of thin $\mathrm{F}$ and $\mathrm{N}$ layers $d_{F, N} \ll \xi_{F, N}$. In this case one can neglect both the derivative on $x$ and nongradient items in Usadel equations (4) and substitute the resulting solutions

$$
\begin{gathered}
\theta_{F, N}(x, y)=\theta_{F, N}(x)-K_{F, N}(x) \frac{d_{F, N}}{\xi_{F, N}^{2}} y+K_{F, N}(x) \frac{y^{2}}{2 \xi_{F, N}^{2}} \\
K_{F, N}(x)=\left\{\frac{\widetilde{\omega}}{\pi T_{c}} \sin \theta_{F, N}-\xi_{F, N}^{2} \frac{\partial^{2}}{\partial x^{2}} \theta_{F, N}\right\} \frac{d_{F, N}}{\xi_{F, N}^{2}}
\end{gathered}
$$

into boundary conditions (5). Then the problem is reduced to the one-dimensional equations for lateral variations of $\theta_{N, F}$ in the $x$-direction:

$$
\zeta_{N, F}^{2} \frac{\partial^{2}}{\partial x^{2}} \theta_{N, F}-\sin \left(\theta_{N, F}-\theta_{N, F}( \pm \infty)\right)=0
$$

where

$$
\theta_{N, F}( \pm \infty)=\arctan \frac{\pi T_{c} \sin \theta_{S}}{\widetilde{\omega} \tilde{\gamma}+\pi T_{c} \cos \theta_{S}}
$$

the decay lengths $\zeta_{N}$ and $\zeta_{F}$ are

$$
\zeta_{N, F}=\xi_{N, F} \sqrt{\frac{\pi T_{c} \tilde{\gamma} \cos \theta_{N, F}( \pm \infty)}{\left(\widetilde{\omega} \tilde{\gamma}+\pi T_{c} \cos \theta_{S}\right)}}
$$

and we have taken for simplicity equal barrier parameters for $\mathrm{F}$ and $\mathrm{N}$

$$
\gamma_{B N} \frac{d_{N}}{\xi_{N}}=\gamma_{B F} \frac{d_{F}}{\xi_{F}} \equiv \widetilde{\gamma}
$$

The general solution of Eq. (9) has the form

$$
\begin{gathered}
\theta_{N, F}(x)=\theta_{N, F}( \pm \infty)+ \\
+4 \arctan \left[\left(\tan \frac{\theta_{N, F}(0)-\theta_{N, F}( \pm \infty)}{4}\right) \exp \left\{\mp \frac{x}{\zeta_{N, F}}\right\}\right],
\end{gathered}
$$

The integration constants $\theta_{N}(0)$ and $\theta_{F}(0)$ in (13) have to be determined from the boundary conditions (6) at $x=0$ and can be found analytically in the limit of large transparency of the FN interface when $\theta(x)$ is continuous at at $x=0$

$$
\theta_{N}(0)=\theta_{F}(0)=\theta(0)
$$

From (11) it follows that the effective decay length in normal metal, $\zeta_{N}$, is a real quantity and equals to $\zeta_{N}=\xi_{N} \sqrt{\widetilde{\gamma}}$ for small $\omega$ and tends to $\zeta_{N}=\xi_{N} \sqrt{\pi T_{c} / \omega}$ with $\omega$ increase. The effective decay length $\zeta_{F}$ in ferromagnet and at low $\omega \ll \Delta, H / \widetilde{\gamma}$ is given by $\zeta_{F}=$ $\xi_{F} \sqrt{\widetilde{\gamma} / \sqrt{1-\widetilde{\gamma}^{2}\left(H / \pi T_{c}\right)^{2}}}$ i. e. it becomes complex for sufficiently strong exchange field $H>\pi T_{c} / \widetilde{\gamma}$.

Below we consider several limiting cases.

Identical $\mathbf{F}$ and $\mathbf{N}$ metals. Assume for simplicity that the $\mathrm{F}$ and $\mathrm{N}$ materials differ by the existence the exchange field in $\mathrm{F}\left(\gamma=1, \xi_{F}=\xi_{N}=\xi\right)$, then from (6) for $\theta(0)$ we have

$$
\theta(0)=2 \arctan \frac{\sin \frac{\theta_{N}(\infty)}{2}+g \sin \frac{\theta_{F}(-\infty)}{2}}{\cos \frac{\theta_{N}(\infty)}{2}+g \cos \frac{\theta_{F}(-\infty)}{2}}, \quad g=\frac{\zeta_{N}}{\zeta_{F}}
$$

Identical $\mathbf{F}$ metals with antiparallel direction of magnetization. The results can be easily generalized to the case of an $\mathrm{S}(\mathrm{F} / \mathrm{F})$ structure with two identical ferromagnetic films having opposite magnetization directions (antiferromagnetic configuration)

$$
\theta(0)=2 \arctan \frac{g^{*} \sin \frac{\theta_{F}^{*}(-\infty)}{2}+g \sin \frac{\theta_{F}(-\infty)}{2}}{g^{*} \cos \frac{\theta_{F}^{*}(-\infty)}{2}+g \cos \frac{\theta_{F}(-\infty)}{2}},
$$

Using the solutions obtained above one can calculate the spatially resolved DoS in $\mathrm{S}(\mathrm{F} / \mathrm{N})$ and $\mathrm{S}(\mathrm{F} / \mathrm{F})$ structures. 


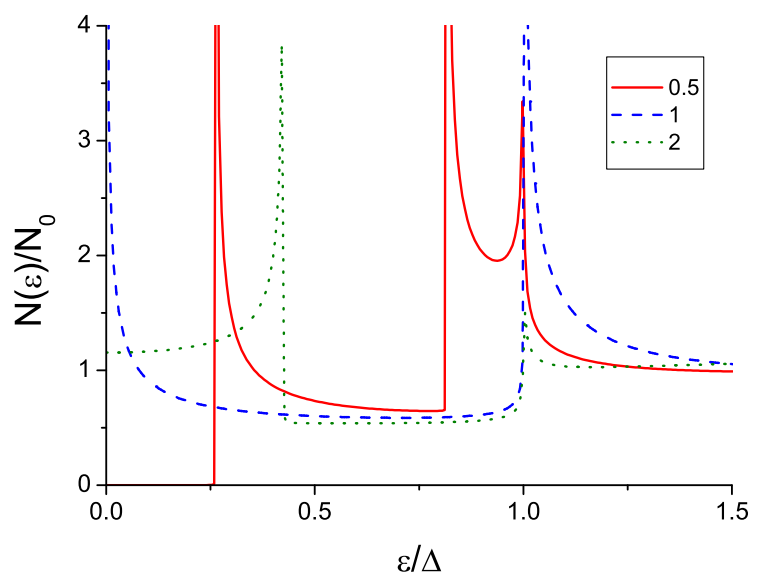

FIG. 2: The total DoS in an SF bilayer for various values of $\widetilde{\gamma} h$ as indicated in the figure.

DoS in $S(F / N)$ and $S(F / F)$ proximity systems. The DoS for each spin direction is given by

$$
N(\varepsilon)=\frac{N_{0}}{2} \operatorname{Re} G(\omega \rightarrow-i \varepsilon+\delta),
$$

where $N_{0}$ is the total DoS for both spins at the Fermi surface in the normal state and $G(\varepsilon-i \delta)=\cos \theta(\varepsilon-i \delta)$ is the retarded Green's function. The total DoS is found by summing over both spin projections i.e. $N^{\text {total }}=$ $N(H)+N(-H)$.

DoS in $\mathrm{N}$ and $\mathrm{F}$ metals far from the $\mathrm{F} / \mathrm{N}$ interface. In a normal metal far from the $\mathrm{F} / \mathrm{N}$ interface $(x=\infty)$ the total DoS is given by

$$
\begin{gathered}
N_{N}(\varepsilon)=N_{0} \operatorname{Re} \frac{\widetilde{\varepsilon}_{N}}{\Omega_{N}}, \quad \Omega_{N}=\sqrt{\widetilde{\varepsilon}_{N}^{2}-\Delta^{2}} \operatorname{sgn}(\varepsilon) \\
\widetilde{\varepsilon}_{N}=\varepsilon\left(1+\widetilde{\gamma} \sqrt{\Delta^{2}-\varepsilon^{2}} / \pi T_{c}\right) .
\end{gathered}
$$

It is well known (see Refs ${ }^{25,26}$ ) that DoS in a $\mathrm{F} / \mathrm{N}$ bilayer has a minigap at $\varepsilon_{g}<\Delta$, which depends on the value of $\widetilde{\gamma}$, and $N_{N}(\varepsilon)$ has the peaks at $\varepsilon=\varepsilon_{g}$ and $\varepsilon=\Delta$. The minigap $\varepsilon_{g}$ characterizes the strength of superconducting correlations induced into $\mathrm{N}$ metal due to the proximity effect.

In SF bilayers, modifications of DoS due to spin splitting of energy levels were investigated in Refs ${ }^{20.21}$. The DoS per spin projection in the $\mathrm{F}$ layer has the form

$$
\begin{gathered}
N_{F \uparrow, F \downarrow}(\varepsilon)=\frac{N_{0}}{2} \operatorname{Re} \frac{\widetilde{\varepsilon}_{F \uparrow, F \downarrow}}{\Omega_{F \uparrow, F \downarrow}}, \\
\Omega_{F \uparrow, F \downarrow}=\sqrt{\widetilde{\varepsilon}_{F \uparrow, F \downarrow}^{2}-\Delta^{2}} \operatorname{sgn}(\varepsilon \mp H), \\
\widetilde{\varepsilon}_{F \uparrow, F \downarrow}=\varepsilon+\widetilde{\gamma}(\varepsilon \mp H) \sqrt{\Delta^{2}-\varepsilon^{2}},
\end{gathered}
$$

which demonstrates the energy renormalization due to the exchange field. In particular, it follows from (18)

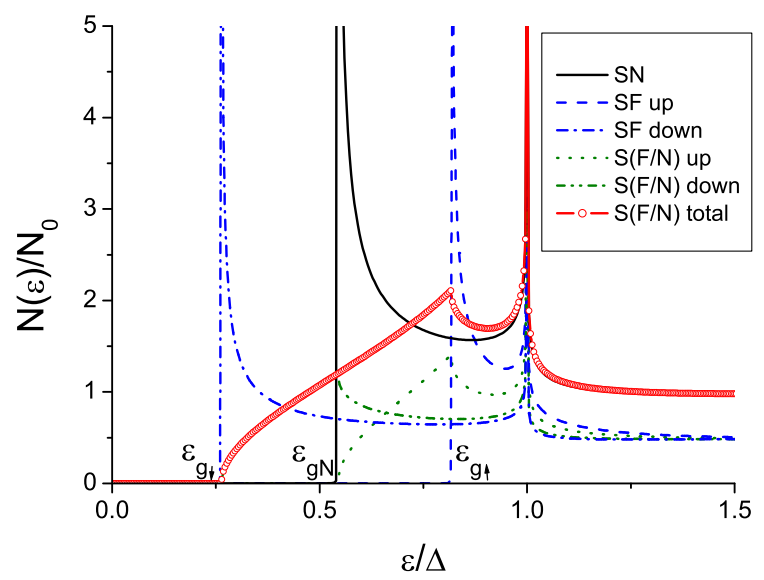

FIG. 3: Spin resolved DoS: comparison of FS, FN and S(FN) for $h \widetilde{\gamma}=0.5$.

that now there are two minigaps in the spectrum $\varepsilon_{g \uparrow}$ and $\varepsilon_{g \downarrow}$ and $\varepsilon_{g \uparrow} \leq \varepsilon_{g} \leq \varepsilon_{g \downarrow}$.

The total DoS in a F/N bilayer $N_{t o t}(\varepsilon)=N_{F \downarrow}(\varepsilon)+$ $N_{F \uparrow}(\varepsilon)$ is shown in Fig.2. It is clearly seen that at $h=H / \pi T_{c}<1 / \widetilde{\gamma}$ there are three peaks in DoS located at $\varepsilon_{g \downarrow}, \varepsilon_{g \uparrow}$ and $\Delta$ respectively. At $h=1 / \widetilde{\gamma}$ the low energy singularity is shifted to the Fermi level and for $h>1 / \widetilde{\gamma}$ the first peak disappears resulting in only two singularities in the DoS at $\varepsilon=\varepsilon_{g \uparrow}$ and $\varepsilon=\Delta$. Note that the total DoS at low energies depends nonmonotonously on $H$ even in a thin F-layer, even though spatial oscillations are absent across the layer. Eq. (18) yields $\left.N_{F \uparrow, F \downarrow}(\varepsilon=0)=\left(N_{0} / 2\right) \operatorname{Re} \widetilde{\gamma} h \operatorname{sgn}(h) / \sqrt{\widetilde{\gamma}^{2} h^{2}-1}\right)$. For $\widetilde{\gamma} h<1$ the total DoS $N(0)=0$ due to the minigap in F while for $\widetilde{\gamma} h \geq 1$ the total low-energy DoS increases sharply, exceeds unity and saturates at $N(0)=N_{0}$ for 1 $\widetilde{\gamma} h \gg 1$.

DoS at the $\mathbf{F} / \mathbf{N}$ interface. At $x=0$ and for identical transport parameters on the $\mathrm{F}$ and $\mathrm{N}$ metals from (14), (16) we obtain

$$
N_{F \uparrow, F \downarrow}(\varepsilon)=\frac{N_{0}}{2} \operatorname{Re} \frac{-i \widetilde{\varepsilon}_{N}-i \widetilde{\varepsilon}_{F \uparrow, F \downarrow}+2 \widetilde{\varepsilon}_{F N}}{\Omega_{F \uparrow, F \downarrow}+\Omega_{N}+2 \sqrt{\Delta^{2}-\widetilde{\varepsilon}_{F N}^{2}}},
$$

where

$$
\widetilde{\varepsilon}_{F N}=\sqrt{\frac{\Omega_{N} \Omega_{F \uparrow, F \downarrow}-\widetilde{\varepsilon}_{N} \widetilde{\varepsilon}_{F \uparrow, F \downarrow}-\Delta^{2}}{2}},
$$

and $\Omega_{N}, \widetilde{\varepsilon}_{N}$ and $\Omega_{F \uparrow, F \downarrow}, \widetilde{\varepsilon}_{F \uparrow, F \downarrow}$ are defined by (17) and (18) respectively.

It follows from Eq. (19) that, similar to the case of the SF bilayer considered above, the minigap exists if $\widetilde{\gamma} h<1$. With increasing exchange field the total DoS at $\varepsilon=0$ becomes nonzero if $\widetilde{\gamma} h>1$ at is given by simple expression

$$
N(0)=N_{0} \sqrt{\widetilde{\gamma}^{2} h^{2}-1} / \widetilde{\gamma} h
$$




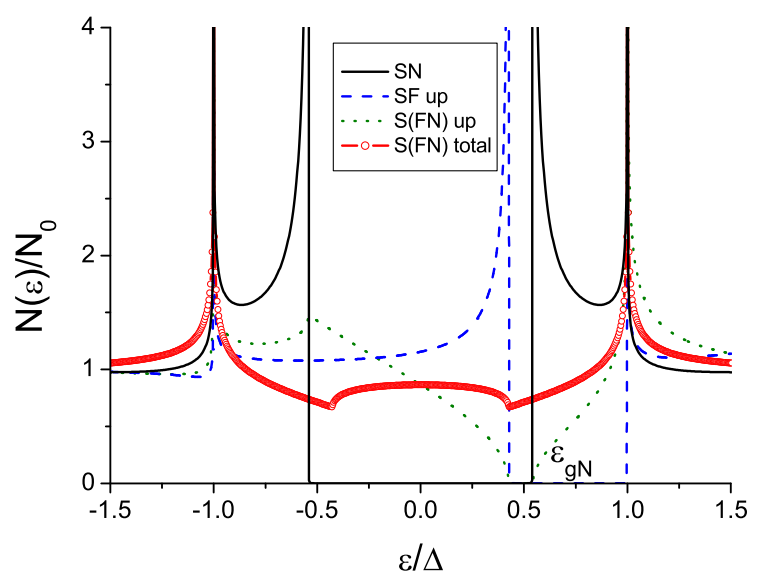

FIG. 4: Spin resolved DoS: comparison of FS, FN and S(FN) for $h \widetilde{\gamma}=2$.

DoS at the $\mathbf{F} / \mathbf{F}$ interface. At $x=0$ we have

$$
\begin{gathered}
N(\varepsilon) / N_{0}=\operatorname{Re} \frac{-2 i \widetilde{\varepsilon}_{N}+2 \widetilde{\varepsilon}_{F F}}{\Omega_{F \uparrow}+\Omega_{F \downarrow}+2 \sqrt{\Delta^{2}-\widetilde{\varepsilon}_{F F}^{2}}} \\
\widetilde{\varepsilon}_{F F}=\sqrt{\frac{\Omega_{F \uparrow} \Omega_{F \downarrow}-\widetilde{\varepsilon}_{F \uparrow} \widetilde{\varepsilon}_{F \downarrow}-\Delta^{2}}{2}}
\end{gathered}
$$

It can be shown that DoS at the $\mathrm{F} / \mathrm{F}$ interface given by Eq.(22) coincides exactly with the total DoS for the F/N interface, $N_{F \uparrow}(\varepsilon)+N_{F \downarrow}(\varepsilon)$, where $N_{F \uparrow}(\varepsilon)$ and $N_{F \downarrow}(\varepsilon)$ are given by Eq. (19). In particular, the minigap exists if $\widetilde{\gamma} h \leq 1$ and at $\widetilde{\gamma} h>1$ the DoS at $\mathrm{F} / \mathrm{F}$ is determined by Eq.(21).

The results of calculations from Eq. 119) at low temperatures $T \ll T_{c}$ are shown in Figs.3,4 for $h \widetilde{\gamma}=0.5$ and $h \widetilde{\gamma}=2$, respectively, together with the DoS for SF $(x \rightarrow-\infty)$ and SN $(x \rightarrow \infty)$ bilayers.

There are four characteristic energies in the system: $\varepsilon_{g \downarrow}, \varepsilon_{g}, \varepsilon_{g \uparrow}$ and $\Delta$. Here $\varepsilon_{g \downarrow}$ is the minigap for the spindown subband $\mathrm{SF}$ bilayer at $x \rightarrow-\infty$. It follows from Eq. (19) that $N_{F \downarrow}(\varepsilon)=0$ at $\varepsilon \leq \varepsilon_{g \downarrow}$ and becomes nonzero at $\varepsilon>\varepsilon_{g \downarrow}$, i.e. $\varepsilon_{g \downarrow}$ is the minigap for the spin-down subband in $\mathrm{S}(\mathrm{FN})$ at $x=0$. However, contrary to $\mathrm{SF}$ case $N_{F \downarrow}(\varepsilon)$ has no peak $\varepsilon=\varepsilon_{g \downarrow}$ but grows continuously from zero value.

For the spin-up subband, the minigap in $N_{F \uparrow}(\varepsilon)$ is not equal to the gap $\varepsilon_{g \uparrow}$ in the spin-up subband in SF bilayer at $x \rightarrow-\infty$. Instead, $N_{F \uparrow}(\varepsilon)$ the gap value is determined by $\varepsilon_{g}$, the minigap in SN bilayer at $x \rightarrow \infty$. The formal reason is that in the interval $\varepsilon \geq \varepsilon_{g}, \Omega_{N}$ becomes an imaginary number and both numerator and denominator in Eq. (19) are complex thus leading to nonzero DoS in this energy range. Similar to the spin-down case, there is no peak in $N_{F \uparrow}(\varepsilon)$ at the gap energy $\varepsilon=\varepsilon_{g}$, while the peak occurs at $\varepsilon=\varepsilon_{g \uparrow}$ (see Fig.3). With further increase

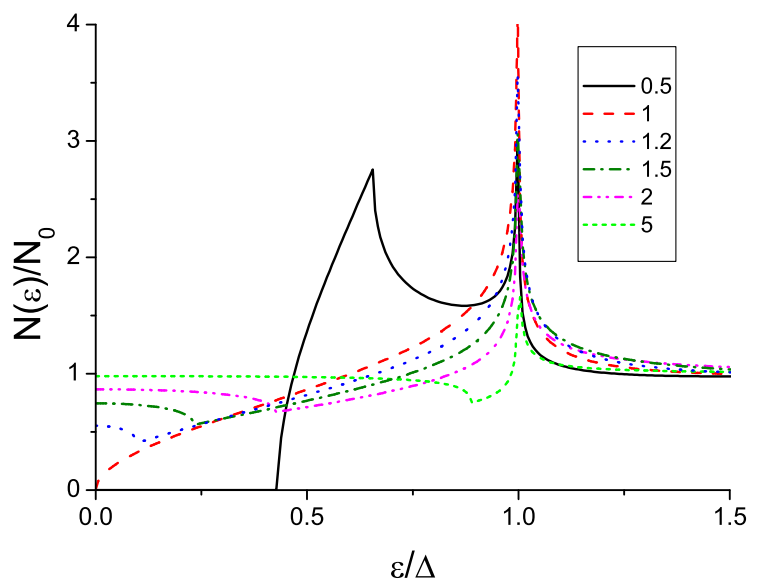

FIG. 5: The total DoS in $\mathrm{S}(\mathrm{FN})$ for various values of $\widetilde{\gamma} h$ as indicated in the figure.

of energy there is a sharp peak in DoS at $\varepsilon=\Delta$ followed by saturation at $N_{0} / 2$ for $\varepsilon \gg \Delta$.

For $h \widetilde{\gamma}>1$ the minigap at $N_{F \downarrow}(\varepsilon)$ vanishes and the structure of DoS becomes different, as illustrated in Fig.4 for the case $h \widetilde{\gamma}=2$. The main qualitative difference from the previous case is that the spin-down and total DoS are gapless for $h \widetilde{\gamma}<1$.

The total DoS at the $\mathrm{F} / \mathrm{N}$ interface at $x=0$ (which coincides with the total DoS in the F/F case), is shown in Fig. 5 for various values of $h \widetilde{\gamma}$. One can see that the gap is closed at $h \widetilde{\gamma}=1$ and the broad zero-energy DoS peak is formed with further increase of $h$ until low-energy states become continuously filled at $h \widetilde{\gamma} \gg 1$.

In conclusion, we have studied theoretically the spatially resolved DoS in the $\mathrm{S}(\mathrm{FN})$ structures and in $\mathrm{S}(\mathrm{FF})$ structures with antiparallel magnetization directions. Analytical solutions were obtained in the case of thin F, N layers which demonstrate the peculiar features of DoS in this system. We have illustrated the results numerically and have studied the dependencies of the minigap and the DoS peak positions on the exchange energy and parameters of the layers.

This work has been supported in part by Russian Ministry of Education and Science and RFBR Grant N 04 0217397-a.
1 A.A.Golubov, M.Yu.Kupriyanov, and E. Il'ichev, Rev. Mod. Phys. 76, 411 (2004).
2 A. I. Buzdin, L. N. Bulaevskii, and S. V. Panyukov, Pis'ma 
Zh. Eksp. Teor. Phys. 35, 147 (1982) [JETP Lett. 35, 178 (1982)].

3 A. I. Buzdin and M. Yu. Kupriyanov, Pis'ma Zh. Eksp. Teor. Phys. 53, 308 (1991) [JETP Lett. 53, 321 (1991)].

4 A. I. Buzdin, B. Vujičić, and M. Yu. Kupriyanov, Zh. Eksp. Teor. Phys. 101, 231 (1992) [Sov. Phys. JETP 74, 124 (1992)].

5 V.V.Ryazanov, V.A.Oboznov, A.Yu.Rusanov, V.V.Veretennikov, A.A.Golubov, J.Aarts, Phys.Rev.Lett., 86, 2427 (2001).

${ }^{6}$ V. V. Ryazanov, V. A. Oboznov, A. V. Veretennikov, and A. Yu. Rusanov, Phys. Rev. B 65, 020501 (2002).

7 O. Bourgeois, P. Gandit, A. Sulpice, J. Chaussy, J. Lesueur, and X. Grison, Phys. Rev. B 63, 064517 (2001).

8 T. Kontos, M. Aprili, J. Lesueur, F. Genet, B. Stephanidis, and R.Boursier, Phys. Rev. Lett. 89, 137007 (2002).

9 C.Surgers, T.Hoss, C.Schonenberger, C.Strunk, Journal of Magnetism and Magnetic Materials, 240, 598 (2002).

10 Y. Blum, A. Tsukernik, M. Karpovski, A. Palevski, Phys. Rev. Lett. (2002).

11 H. Sellier, C. Baraduc, F. Lefloch, and R. Calemczuk, Phys. Rev. B 68, 054531 (2003).

12 R.Mints, Phys. Rev. B 57, R3221 (1998); R. G. Mints and I. Papiashvili, Phys. Rev. B 64, 134501 (2001).

13 A. I. Buzdin and A.E. Koshelev, Phys. Rev. B 67, 220504 (2003).
14 H. Hilgenkamp, Ariando, H.J.H. Smilde, D.H.A. Blank, G. Rijnders, H. Rogalla, J.R. Kirtley and C.C. Tsuei, Nature 422, 50 (2003).

15 T. Kontos, M. Aprili, J. Lesueur, and X. Grison, Phys. Rev. Lett. 86, 304 (2001).

16 A.I. Buzdin, Phys. Rev. B 62, 11377 (2000).

17 I.Baladie and A. Buzdin, Phys. Rev. B 64, 224514 (2001).

18 M. Zareyan, W. Belzig, and Yu. V. Nazarov, Phys. Rev. Lett. 86, 308 (2001); Phys. Rev. B 65, 184505 (2002).

19 F. S. Bergeret, A. F. Volkov, and K. B. Efetov, Phys. Rev. B 65, 134505 (2002).

${ }^{20}$ V. N. Krivoruchko and E. A. Koshina, Phys. Rev. B 66, 014521 (2002).

21 A. A. Golubov, M. Yu. Kupriyanov, and Ya. V. Fominov, Pis'ma Zh. Eksp. Teor. Fiz. 75, 223 (2002) [JETP Lett. 75, 190 (2002)].

22 D.Huertas-Hernando and Yu.V.Nazarov, preprint cond-mat/0404622

23 K.D.Usadel, Phys.Rev.Lett., 25, 507 (1970).

24 M.Yu.Kupriyanov, V.F.Lukichev, Zn.Eksp.Teor.Phys. 94, 139 (1988) [Sov.Phys.JETP. 67, 1163 (1988)].

25 W.L.McMillan, Phys. Rev. 175, 537 (1967).

26 A.A.Golubov, M.Yu.Kupriyanov, Zh.Eksp.Teor.Phys. 96, 1420 (1989) [Sov.Phys.JETP. 69, 805 (1989)]. 